\title{
C*-SEMINORMS ON PARTIAL *-ALGEBRAS: AN OVERVIEW
}

\author{
CAMILLO TRAPANI \\ Dipartimento di Matematica ed Applicazioni, Università di Palermo \\ 90123 Palermo, Italy \\ E-mail: trapani@unipa.it
}

\begin{abstract}
The main facts about unbounded $\mathrm{C}^{*}$-seminorms on partial ${ }^{*}$-algebras are reviewed and the link with the representation theory is discussed. In particular, starting from the more familiar case of ${ }^{*}$-algebras, we examine $\mathrm{C}^{*}$-seminorms that are defined from suitable families of positive linear or sesquilinear forms, mimicking the construction of the Gelfand seminorm for Banach ${ }^{*}$-algebras. The admissibility of these forms in terms of the (unbounded) $\mathrm{C}^{*}$-seminorms they generate is characterized.
\end{abstract}

1. Introduction. As is well known, $\mathrm{C}^{*}$-seminorms give a deep insight into the structure of Banach *-algebras [13, Ch. V.39] in particular for the close relationship they have with positive linear functionals [14]. Their role is also crucial for the study of *-representations, since through them the representation theory of $\mathrm{C}^{*}$-algebras can be invoked and provides a full amount of information on the representations of the starting algebra. To be more precise, if $\mathfrak{A}_{0}$ is a ${ }^{*}$-algebra and $p$ a $\mathrm{C}^{*}$-seminorm on $\mathfrak{A}_{0}$, then every *-representation of the Hausdorff completion of $\left(\mathfrak{A}_{0}, p\right)$ defines a bounded ${ }^{*}$-representation of $\mathfrak{A}_{0}$ in Hilbert space [12].

The notion of $\mathrm{C}^{*}$-seminorms has been first considered by Fell [16] and Effros [15] and researches on this topic have been undertaken in several different directions, according to the various situations where they arise. Some geometric aspects of the collection of all $\mathrm{C}^{*}$-seminorms defined on a Banach *-algebra are summarized in [13, Ch. V.39]. Also extensions of this notion to at least two more general cases have been considered. The first one, $[25,12]$ consists in considering unbounded $\mathrm{C}^{*}$-seminorms on a ${ }^{*}$-algebra $\mathfrak{A}_{0}$, i.e. $\mathrm{C}^{*}$-seminorms $p$ defined only on a ${ }^{*}$-subalgebra $D(p)$ of $\mathfrak{A}_{0}$. The interest for non-everywhere defined $\mathrm{C}^{*}$-seminorms comes from the fact that they appear in many mathematical $[19,25]$ and physical applications $[1,24]$. In particular, Yood studied [25] $\mathrm{C}^{*}$-seminorms on a ${ }^{*}$-algebra $\mathfrak{A}_{0}$ that can be defined via a family $\mathcal{F}$ of positive linear func-

2000 Mathematics Subject Classification: Primary 46K05.

The paper is in final form and no version of it will be published elsewhere. 
tionals on $\mathfrak{A}_{0}$. These $\mathrm{C}^{*}$-seminorms, whose definition is strongly inspired by the Gelfand seminorm on a Banach *-algebra, are, in general, not everywhere defined and for this reason they have been called unbounded. Yood also gave a characterization of $\mathrm{C}^{*}$-seminorms defined by admissible positive linear functionals. The importance of admissibility relies on the fact that the Gelfand-Naimark-Segal (GNS) construction based on an admissible form produces a bounded representation. The role of unbounded $\mathrm{C}^{*}$-seminorms for the representation theory of a ${ }^{*}$-algebra has been studied by Bhatt, Inoue and Ogi in [12].

A second generalization of the theory of $\mathrm{C}^{*}$-seminorms is obtained by enlarging the environment where to consider them replacing *-algebras with partial ${ }^{*}$-algebras. A first study in this direction was made in [5], where unbounded $\mathrm{C}^{*}$-seminorms on partial * algebras [3] were studied with the aim of extending some results of representation theory obtained in [12] in the case of *-algebras.

Recently a study on the possibility of extending Yood's approach to the partial algebraic setting has been undertaken [22] for the case of quasi *-algebras and in [23] for general partial *-algebras. In both cases positive linear functional are systematically replaced with positive sesquilinear forms, due to the lack of an everywhere defined multiplication. In particular, in the case of partial *-algebras, a relevant role is played by a special kind of non-everywhere defined sesquilinear forms called biweights: they are exactly the kind of forms that allow a GNS construction for partial *-algebras $[2,3]$.

In this paper we will shortly review all the subject of (unbounded) $\mathrm{C}^{*}$-seminorms, focusing our attention on those constructed by means of families of positive linear or sesquilinear forms, starting from the very familiar case of Banach *-algebras, then we survey Yood's results for the case of a *-algebra with no topology given a priori and then we summarize the main results concerning the generalization to partial *-algebras.

Before going forth, we give, for the reader's convenience, the definitions of partial *-algebra and quasi *-algebra that are needed in the sequel.

A partial *-algebra is a complex vector space $\mathfrak{A}$, endowed with an involution $x \mapsto x^{*}$ (that is, a bijection such that $x^{* *}=x$ ) and a partial multiplication defined by a set $\Gamma \subset \mathfrak{A} \times \mathfrak{A}$ (a binary relation) such that:

(i) $(x, y) \in \Gamma$ implies $\left(y^{*}, x^{*}\right) \in \Gamma$;

(ii) $\left(x, y_{1}\right),\left(x, y_{2}\right) \in \Gamma$ implies $\left(x, \lambda y_{1}+\mu y_{2}\right) \in \Gamma, \forall \lambda, \mu \in \mathbb{C}$;

(iii) for any $(x, y) \in \Gamma$, there is defined a product $x \cdot y \in \mathfrak{A}$, which is distributive w.r. to the addition and satisfies the relation $(x \cdot y)^{*}=y^{*} \cdot x^{*}$.

We shall assume the partial *-algebra $\mathfrak{A}$ contains a unit $e$, i.e., $e^{*}=e,(e, x) \in \Gamma, \forall x \in$ $\mathfrak{A}$, and $e \cdot x=x \cdot e=x, \forall x \in \mathfrak{A}$. (If $\mathfrak{A}$ has no unit, it may always be embedded into a larger partial *-algebra with unit, in the standard fashion.)

Given the defining set $\Gamma$, spaces of multipliers are defined in the obvious way:

$$
\begin{aligned}
(x, y) \in \Gamma & \Leftrightarrow x \in L(y) \text { or } x \text { is a left multiplier of } y \\
& \Leftrightarrow y \in R(x) \text { or } y \text { is a right multiplier of } x .
\end{aligned}
$$

One of the most relevant examples of partial *-algebras is provided by a notable family of unbounded operators in Hilbert space $\mathcal{H}$. In fact, let $\mathcal{D}$ be a dense domain in $\mathcal{H}$; we 
define

$$
\mathcal{L}^{\dagger}(\mathcal{D}, \mathcal{H})=\left\{A \text { closable }: D(A)=\mathcal{D} ; \quad D\left(A^{*}\right) \supset \mathcal{D}\right\}
$$

The vector space $\mathcal{L}^{\dagger}(\mathcal{D}, \mathcal{H})$ becomes a partial *-algebra with the involution $A \mapsto A^{\dagger}=$ $A^{*} \Gamma_{\mathcal{D}}$ and weak partial multiplication defined as follows:

$$
\begin{aligned}
& (A, B) \in \Gamma \Leftrightarrow B \mathcal{D} \subset D\left(A^{\dagger *}\right) \text { and } A^{\dagger} \mathcal{D} \subset D\left(B^{*}\right) \\
& (A \square B) \xi:=A^{\dagger *} B \xi, \quad \forall \xi \in \mathcal{D} .
\end{aligned}
$$

A quasi ${ }^{*}$-algebra $\left(\mathfrak{A}, \mathfrak{A}_{0}\right)$ is a partial *-algebra where $\mathfrak{A}_{0}$ is a ${ }^{*}$-algebra $\mathfrak{A}_{0} \subset \mathfrak{A}$ and

$$
\Gamma=\left\{(a, b) \in \mathfrak{A} \times \mathfrak{A}: a \in \mathfrak{A}_{0} \text { or } b \in \mathfrak{A}_{0}\right\} .
$$

The definition of quasi *-algebra was originally given by Lassner $[17,18])$ independently of that of partial *-algebra. Following [20], if $\left(\mathfrak{A}, \mathfrak{A}_{0}\right)$ is a quasi *-algebra, we shall always assume that $\mathfrak{A}$ is an $\mathfrak{A}_{0}$-bimodule.

The most typical example of a quasi *-algebra is provided by the completion $\mathfrak{A} \equiv \widehat{\mathfrak{A}_{0}}$ of a locally convex *-algebra $\mathfrak{A}_{0}[\tau]$ whose multiplication is not jointly continuous.

Full details on partial *-algebras and their representation theory can be found in [3].

\section{The case of $*$-algebras}

Definition 1 . Let $\mathfrak{A}_{0}$ be a ${ }^{*}$-algebra with unit $e$. A seminorm $p$ on $\mathfrak{A}_{0}$ is called a $\mathrm{C}^{*}$ seminorm if

(1) $p(a b) \leq p(a) p(b)$ for all $a, b \in \mathfrak{A}_{0}$;

(2) $p\left(a^{*} a\right)=p(a)^{2}$ for each $a \in \mathfrak{A}_{0}$.

Of course, (1) and (2) imply that $p\left(a^{*}\right)=p(a)$ for each $a \in \mathfrak{A}_{0}$ and if, $p \neq 0, p(e)=1$. From a beautiful result of Sebestyén [21] it follows that (2) implies (1).

EXAmple 1 (Normed *algebras). It is well-known that $\mathrm{C}^{*}$-seminorms can be used to get information on the structure of a normed or Banach *-algebra [13]. We collect here, just as example, some very basic facts for this case.

If $\mathfrak{A}_{0}$ is a Banach *-algebra with unit $e$, then each positive linear functional $\omega$ is continuous and $\|\omega\|=\omega(e)$. This fact allows us to construct the so-called GelfandNaimark seminorm $p$ on $\mathfrak{A}_{0}$ by putting

$$
p(a)=\sup _{\omega \in \mathcal{S}\left(\mathfrak{A}_{0}\right)} \omega\left(a^{*} a\right)^{1 / 2}, \quad a \in \mathfrak{A}_{0} .
$$

where $\mathcal{S}\left(\mathfrak{A}_{0}\right)$ denotes the set of all positive linear functionals $\omega$ on $\mathfrak{A}_{0}$ with $\omega(e)=1$. One has: $p(a) \leq\|a\|$ for every $a \in \mathfrak{A}_{0}$. If $\omega$ is a positive linear functional on $\mathfrak{A}_{0}$ and $x \in \mathfrak{A}_{0}$, then the linear functional $\omega_{x}$ defined by $\omega_{x}(a)=\omega\left(x^{*} a x\right)$ is also positive and if $\omega\left(x^{*} x\right)=1$ then $\omega_{x} \in \mathcal{S}\left(\mathfrak{A}_{0}\right)$. Using this fact, that as we shall see will be the key for the construction of Gelfand-like seminorms in general *-algebras [cfr. Definition 2], one can prove that actually $(1)$ defines a $\mathrm{C}^{*}$-seminorm on $\mathfrak{A}_{0}$. In general, $p$ is not a norm; i.e. there may exist non-zero elements $a \in \mathfrak{A}_{0}$ such that $p(a)=0$. Let

$$
\mathfrak{N}_{p}=\left\{b \in \mathfrak{A}_{0}: p(b)=0\right\} .
$$

Then it is easily seen that $\mathfrak{N}_{p}$ is a closed *-ideal of $\mathfrak{A}_{0}$. The quotient $\mathfrak{A}_{0} / \mathfrak{N}_{p}$ is a $\mathrm{C}^{*}$ algebra, with norm $\|[a]\|=p(a)$. The set $\mathfrak{N}_{p}$ is nothing but the ${ }^{*}$-radical $\mathfrak{R}^{*}$ of the 
Banach *-algebra $\mathfrak{A}_{0}$ (i.e., the intersection of the kernels of all *-representations of $\mathfrak{A}_{0}$ ) and so ${ }^{*}$-semisimplicity of $\mathfrak{A}_{0}$ corresponds to $\mathfrak{N}_{p}=\{0\}$. If $\mathfrak{A}_{0}$ is a $C^{*}$-algebra, for each $a \in \mathfrak{A}_{0}$, there exists a positive linear functional $\omega$ with $\omega(e)=1$ such that $\omega\left(a^{*} a\right)=\|a\|^{2}$. This then leads to the well-known Gelfand's characterization of the norm of a $\mathrm{C}^{*}$-algebra:

$$
\|a\|=p(a)=\sup _{\omega \in \mathcal{S}\left(\mathfrak{A}_{0}\right)} \omega\left(a^{*} a\right)^{1 / 2}, \quad \forall a \in \mathfrak{A}_{0} .
$$

Gelfand-like seminorms on a *-algebra where no topology is given a priori have been considered by B. Yood [25]. We briefly review his main results.

Definition 2. Let $\mathfrak{A}_{0}$ be ${ }^{*}$-algebra. A family $\mathcal{F}$ of positive linear functionals on $\mathfrak{A}_{0}$ is called balanced if, for each $x \in \mathfrak{A}_{0}$ and $\omega \in \mathcal{F}$, the positive linear functional $\omega_{x}$ defined by

$$
\omega_{x}(a)=\omega\left(x^{*} a x\right), \quad a \in \mathfrak{A}_{0}
$$

also belongs to $\mathcal{F}$.

If $\mathcal{F}$ is a balanced family of positive linear functionals, then one can define:

$$
\mathcal{D}\left(p_{\mathcal{F}}\right)=\left\{a \in \mathfrak{A}_{0}: \sup _{\omega \in \mathcal{F}_{s}} \omega\left(a^{*} a\right)<\infty\right\}
$$

where $\mathcal{F}_{s}=\{\omega \in \mathcal{F}: \omega(a)=e\}$ and

$$
p_{\mathcal{F}}(a)^{2}=\sup _{\omega \in \mathcal{F}_{s}} \omega\left(a^{*} a\right) .
$$

Then $\mathcal{D}\left(p_{\mathcal{F}}\right)$ is a ${ }^{*}$-subalgebra of $\mathfrak{A}_{0}$ and $p_{\mathcal{F}}$ is an unbounded $\mathrm{C}^{*}$-seminorm on $\mathfrak{A}_{0}$.

Definition 3. A positive linear functional $\omega$ on $\mathfrak{A}_{0}$ is called admissible if, for every $a \in \mathfrak{A}_{0}$, there exists $\gamma_{a}>0$ such that

$$
\left|\omega\left(x^{*} a^{*} a x\right)\right| \leq \gamma_{a} \omega\left(x^{*} x\right), \quad \forall x \in \mathfrak{A}_{0} .
$$

Admissibility is a relevant property of positive linear functionals, since it is indeed equivalent to the boundedness of the corresponding GNS representation.

Let $q$ be a seminorm on $\mathfrak{A}_{0}$ and $\omega$ a linear functional on $\mathfrak{A}_{0}$. Then $\omega$ is said to be continuous with respect to $q$, or, simply, $q$-continuous if there exists $\gamma>0$ such that:

$$
|\omega(a)| \leq \gamma q(a), \quad \forall a \in \mathfrak{A}_{0} .
$$

Admissibility is characterized by the following

Proposition 4. A state $\omega$ on $\mathfrak{A}_{0}$ is admissible if, and only if, there is a submultiplicative seminorm $q$ on $\mathfrak{A}_{0}$ such that $\omega$ is $q$-continuous.

Now let us suppose that a $\mathrm{C}^{*}$-seminorm on $\mathfrak{A}_{0}$ is given; it is natural to ask what the relationship between $q$ and the $\mathrm{C}^{*}$-seminorm defined by the family of all $q$-continuous positive linear functionals on $\mathfrak{A}_{0}$ is.

TheOREm 5. Let $q$ be a $C^{*}$-seminorm on $\mathfrak{A}_{0}$ and $\mathcal{F}$ the set of all $q$-continuous positive functionals on $\mathfrak{A}_{0}$. Then $\mathcal{D}\left(p_{\mathcal{F}}\right)=\mathfrak{A}_{0}$ and

$$
q(x)^{2}=\sup _{\omega \in \mathcal{F}_{s}} \omega\left(a^{*} a\right), \quad \forall a \in \mathfrak{A}_{0} .
$$


The following statement characterizes the existence of a maximal $\mathrm{C}^{*}$-seminorm $q$ on $\mathfrak{A}_{0}$, i.e., $q(x) \geq p(x)\left(x \in \mathfrak{A}_{0}\right)$ for any other $\mathrm{C}^{*}$-seminorm $p$ on $\mathfrak{A}_{0}$.

THEOREM 6. Let $q$ be a $C^{*}$-seminorm and $\mathcal{F}$ the set of all $q$-continuous positive functionals on $\mathfrak{A}_{0}$. Then $q$ is a maximal $C^{*}$-seminorm if, and only if, $\mathcal{F}$ coincides with the set of all admissible positive linear functionals on $\mathfrak{A}_{0}$.

Some of Yood's results have been extended to *-algebras without unit by Bhatt, Inoue and Ogi in [12], where also *-representation induced by unbounded $\mathrm{C}^{*}$-seminorms have been studied. The same authors in $[9,11,12]$ have also studied the role that un-

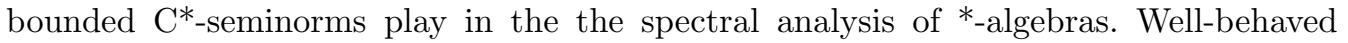
*-representations defined by unbounded $\mathrm{C}^{*}$-seminorms are analyzed in [8]. Several examples can also be found in these papers. Since we shall discuss biweights, let it be noted that admissibility of weights and quasi-weights on a *algebra with very many applications is discussed in [10].

3. The case of a partial *-algebra. In this section we will overview the main facts concerning unbounded $\mathrm{C}^{*}$-seminorms on partial ${ }^{*}$-algebras. We first consider the interplay between $\mathrm{C}^{*}$-seminorms and the representation theory of partial ${ }^{*}$-algebras and then we show how Yood's approach for *-algebras can be extended to the present case. For a more complete discussion we refer to $[5,23]$.

A mapping $p$ of a (partial) *-subalgebra $\mathcal{D}(p)$ of $\mathfrak{A}$ into $\mathbb{R}^{+}$is said to be an unbounded $m^{*}$-(semi)norm on $\mathfrak{A}$ if

(i) $p$ is a (semi) norm on $\mathcal{D}(p)$;

(ii) $p\left(x^{*}\right)=p(x), \quad \forall x \in \mathcal{D}(p)$;

(iii) $p(x y) \leq p(x) p(y), \quad \forall x, y \in \mathcal{D}(p)$ such that $x \in L(y)$.

An unbounded $m^{*}$-(semi)norm $p$ on $\mathfrak{A}$ is said to be an unbounded $\mathrm{C}^{*}$-(semi)norm if

(iv) $p\left(x^{*} x\right)=p(x)^{2}, \quad \forall x \in \mathcal{D}(p)$ such that $x^{*} \in L(x)$.

An unbounded $m^{*}$-(semi)norm (resp. $\mathrm{C}^{*}$-(semi)norm) on $\mathfrak{A}$ is said to be an $m^{*}$ (semi)norm (resp. $C^{*}-($ semi)norm) if $\mathcal{D}(p)=\mathfrak{A}$.

An (unbounded) $m^{*}$-seminorm $p$ on $\mathfrak{A}$ is said to have Property (B) if it satisfies the following basic density-condition:

(B) $\quad R(\mathfrak{A}) \cap \mathcal{D}(p)$ is dense in $\mathcal{D}(p)$ with respect to $p$.

Let $p$ be an unbounded $\mathrm{C}^{*}$-seminorm with property $(\mathrm{B})$. Let us denote with $\hat{\mathfrak{A}}$ the set of all $p$ - Cauchy sequences and in this set we introduce the equivalence relation

$$
\left\{a_{n}\right\} \sim\left\{b_{n}\right\} \Leftrightarrow \lim _{n \rightarrow \infty} p\left(a_{n}-b_{n}\right)=0 .
$$

Then, as shown in $[5,3]$, the quotient $\mathfrak{A}_{p} \equiv \widehat{\mathfrak{A}} / \sim$ is a $\mathrm{C}^{*}$-algebra. If $a \in \mathfrak{A}$, we put $\tilde{a}=\left(a_{n}\right)$ with $a_{n}=a$ for every $n \in \mathbb{N}$.

This fact is very relevant for our proposes, since a $\mathrm{C}^{*}$-algebra has plenty of *-representations (and also a faithful one, by the Gelfand-Naimark theorem). These *-representations can be used to construct (quasi) *-representations of $\mathfrak{A}$ that depend in crucial 
way on the algebra

$$
\mathcal{N}_{p}=\{x \in \mathcal{D}(p) \cap R(\mathfrak{A}) ; a x \in \mathcal{D}(p), \forall a \in \mathfrak{A}\} .
$$

We give an outline of the construction.

For any *-representation $\Pi_{p}$ of $\mathfrak{A}_{p}$ we put

$$
\pi_{p}^{0}(x)=\Pi_{p}(\tilde{x}), \quad x \in \mathcal{D}(p) .
$$

where $\tilde{x}$ denotes any sequence $p$-converging to $x$. Then $\pi_{p}^{0}$ is a ${ }^{*}$-representation of $\mathcal{D}(p)$ on $\mathcal{H}_{\Pi_{p}}$.

Of course, what we want to get is a ${ }^{*}$-representation of the whole $\mathfrak{A}$. Then, we begin with defining the domain $\mathcal{D}\left(\pi_{p}\right)$ as the linear span of the set

$$
\left\{\Pi_{p}\left((x y)^{\sim}\right) \xi ; x, y \in \mathfrak{N}_{p}, \xi \in \mathcal{H}_{\Pi_{p}}\right\},
$$

where $\mathcal{H}_{\pi_{p}}$ is the closure of $\mathcal{D}\left(\pi_{p}\right)$ in $\mathcal{H}_{\Pi_{p}}$. Next we define

$$
\pi_{p}(a)\left(\sum_{k} \Pi_{p}\left(\left(x_{k} y_{k}\right)^{\sim}\right) \xi_{k}\right)=\sum_{k} \Pi_{p}\left(\left(a x_{k}\right)^{\sim} \widetilde{y_{k}}\right) \xi_{k}, \quad a \in \mathfrak{A}, \sum_{k} \Pi_{p}\left(\left(x_{k} y_{k}\right)^{\sim}\right) \xi_{k} \in \mathcal{D}\left(\pi_{p}\right) .
$$

Proposition 7. $\pi_{p}$ is a linear map of $\mathfrak{A}$ into $\mathcal{L}^{\dagger}\left(\mathcal{D}\left(\pi_{p}\right), \mathcal{H}_{\pi_{p}}\right)$ satisfying the following properties:

(i) $\pi_{p}\left(a^{*}\right)=\pi_{p}(a)^{\dagger}, \quad \forall a \in \mathfrak{A}$;

(ii) $\pi_{p}(a x)=\pi_{p}(a) \square \pi_{p}(x), \quad \forall a \in \mathfrak{A}, \forall x \in R(\mathfrak{A})$;

(iii) $\left\|\overline{\pi_{p}(x)}\right\| \leq p(x), \quad \forall x \in \mathcal{D}(p)$. Furthermore, if $\pi_{p}$ is faithful then $\left\|\overline{\pi_{p}(x)}\right\|=p(x), \quad \forall x \in \mathfrak{A}$.

A linear map $\pi$ of $\mathfrak{A}$ into $\mathcal{L}^{\dagger}\left(\mathcal{D}\left(\pi_{p}\right), \mathcal{H}_{\pi_{p}}\right)$ satisfying (i) and (ii) above is called a quasi ${ }^{*}$-representation. In order to obtain a true ${ }^{*}$-representation, some additional condition on $p$ is required.

The unbounded $\mathrm{C}^{*}$-seminorm $p$ is called

- finite if $\mathcal{N}_{p}=\mathcal{D}(p)$;

- semifinite if $\mathcal{N}_{p}$ is $p$-dense in $\mathcal{D}(p)$.

TheOREM 8. Let $\mathfrak{A}$ be a partial ${ }^{*}$-algebra and $p$ a semifinite $C^{*}$-seminorm on $\mathfrak{A}$. Then every quasi ${ }^{*}$-representation $\pi_{p}$ induced by $p$ is a bounded *-representation.

REMARK 9. One can also reverse the point of view: given a ${ }^{*}$-representation $\pi$ of a partial *-algebra, it is possible to construct an unbounded $\mathrm{C}^{*}$-seminorm $r_{\pi}$ on $\mathfrak{A}$. Following the same steps as before, we can also build up a ${ }^{*}$-representation $\pi_{r_{\pi}}^{N}$ called natural. The relationship between $\pi_{r_{\pi}}^{N}$ and the ${ }^{*}$-representation $\pi$ where we had started from has been investigated in [5].

3.1. Constructing $C^{*}$-seminorms from biweights. The possibility of constructing $\mathrm{C}^{*}$ seminorms is closely linked to the GNS representation determined by positive linear functionals. In the case of partial *-algebras a GNS contruction is possible starting from a particular class of positive sesquilinear forms called biweights that we define below. 
Let $\varphi$ be a positive sesquilinear form on $\mathcal{D}(\varphi) \times \mathcal{D}(\varphi)$, where $\mathcal{D}(\varphi)$ is a subspace of $\mathfrak{A}$. Then we have

$$
\begin{aligned}
\varphi(x, y) & =\overline{\varphi(y, x)}, \quad \forall x, y \in \mathcal{D}(\varphi) \\
|\varphi(x, y)|^{2} & \leq \varphi(x, x) \varphi(y, y), \quad \forall x, y \in \mathcal{D}(\varphi) .
\end{aligned}
$$

We put

$$
N_{\varphi}=\{x \in \mathcal{D}(\varphi) ; \varphi(x, x)=0\}
$$

By (3) we have

$$
N_{\varphi}=\{x \in \mathcal{D}(\varphi) ; \varphi(x, y)=0, \quad \forall y \in \mathcal{D}(\varphi)\}
$$

and so $N_{\varphi}$ is a subspace of $\mathcal{D}(\varphi)$ and the quotient space $\mathcal{D}(\varphi) / N_{\varphi} \equiv\left\{\lambda_{\varphi}(x) \equiv x+\right.$ $\left.N_{\varphi} ; x \in \mathcal{D}(\varphi)\right\}$ is a pre-Hilbert space with respect to the inner product $\left(\lambda_{\varphi}(x) \mid \lambda_{\varphi}(y)\right)=$ $\varphi(x, y), x, y \in \mathcal{D}(\varphi)$. We denote by $\mathcal{H}_{\varphi}$ the Hilbert space obtained by the completion of $\mathcal{D}(\varphi) / N_{\varphi}$.

DeFinition 10. Let $\varphi$ be a positive sesquilinear form on $\mathcal{D}(\varphi) \times \mathcal{D}(\varphi)$. A subspace $B(\varphi)$ of $\mathcal{D}(\varphi)$ is said to be a core for $\varphi$ if

(i) $B(\varphi) \subset R(\mathfrak{A})$;

(ii) $\{a x ; a \in \mathfrak{A}, x \in B(\varphi)\} \subset \mathcal{D}(\varphi)$;

(iii) $\lambda_{\varphi}(B(\varphi))$ is dense in $\mathcal{H}_{\varphi}$;

(iv) $\varphi(a x, y)=\varphi\left(x, a^{*} y\right), \quad \forall a \in \mathfrak{A}, \forall x, y \in B(\varphi)$;

(v) $\varphi\left(a^{*} x, b y\right)=\varphi(x,(a b) y), \quad \forall a \in L(b), \forall x, y \in B(\varphi)$.

We denote by $\mathcal{B}_{\varphi}$ the set of all cores $B(\varphi)$ for $\varphi$.

Definition 11. A positive sesquilinear form $\varphi$ on $\mathcal{D}(\varphi) \times \mathcal{D}(\varphi)$ such that $\mathcal{B}_{\varphi} \neq \emptyset$ is called a biweight on $\mathfrak{A}$.

For the sake of completeness, we outline the GNS construction for a biweight. Let $\varphi$ be a biweight on $\mathfrak{A}$ with a core $B(\varphi)$. We put

$$
\pi_{\varphi}^{\circ}(a) \lambda_{\varphi}(x)=\lambda_{\varphi}(a x), \quad a \in \mathfrak{A}, x \in B(\varphi) .
$$

Then it follows from (3) and (iii) of Definition 10 that the biweight $\varphi$ satisfies the condition:

$$
\varphi(x, x)=0 \text { for } x \in \mathfrak{B} \text { implies } \varphi(a x, a x)=0 \quad \forall a \in \mathfrak{A} .
$$

Thus $\pi_{\varphi}^{\circ}(a)$ is a well-defined linear operator of $\lambda_{\varphi}(B(\varphi))$ into $\mathcal{H}_{\varphi}$. Furthermore, it follows from (iv) and (v) of Definition 10 that $\pi_{\varphi}^{\circ}$ is a ${ }^{*}$-representation of $\mathfrak{A}$. We denote by $\pi_{\varphi}^{B}$ the closure of $\pi_{\varphi}^{\circ}$. Then the triple $\left(\pi_{\varphi}^{B}, \lambda_{\varphi}, \mathcal{H}_{\varphi}\right)$ is called the GNS construction for the biweight $\varphi$ on $\mathfrak{A}$ with the core $B(\varphi)$.

Definition 12 . Let $\mathcal{F}$ be a family of biweights on $\mathfrak{A}$ and $\mathfrak{B}$ a subspace of $R \mathfrak{A}$. We say that $\mathcal{F}$ is a $\mathfrak{B}$-regular family if $\mathfrak{B}$ is a common core for all elements of $\mathcal{F}$.

If $\mathcal{F}$ is $\mathfrak{B}$-regular we put, for $a \in \mathfrak{A}$ :

$$
p_{\mathcal{F}}(a)=\sup \left\{\varphi(a x, a x)^{1 / 2}, \varphi \in \mathcal{F}, x \in \mathfrak{B}, \varphi(x, x)=1\right\}
$$

and

$$
\mathcal{D}\left(p_{\mathcal{F}}\right)=\left\{a \in \mathfrak{A} ; p_{\mathcal{F}}(a)<\infty\right\}
$$


We also define:

$$
q_{\mathcal{F}}(a)=\sup \left\{\left|\varphi\left(a x_{1}, x_{2}\right)\right|, \varphi \in \mathcal{F}, x_{1}, x_{2} \in \mathfrak{B}, \varphi\left(x_{1}, x_{1}\right)=1, \varphi\left(x_{2}, x_{2}\right)=1\right\}
$$

and

$$
\mathcal{D}\left(q_{\mathcal{F}}\right)=\left\{a \in \mathfrak{A} ; q_{\mathcal{F}}(a)<\infty\right\} .
$$

Using the Schwarz inequality, we easily prove that:

$$
\mathcal{D}\left(p_{\mathcal{F}}\right) \subseteq \mathcal{D}\left(q_{\mathcal{F}}\right) \quad \text { and } \quad q_{\mathcal{F}}(a) \leq p_{\mathcal{F}}(a), \quad \forall a \in \mathcal{D}\left(p_{\mathcal{F}}\right)
$$

With the help of (iii) of Definition 10, one can prove that

$$
q_{\mathcal{F}}(a)=\sup \{|\varphi(a x, y)|, \varphi \in \mathcal{F}, x \in \mathfrak{B}, \varphi(x, x)=1, y \in \mathcal{D}(\varphi), \varphi(y, y)=1\} .
$$

From this equality, one gets that, for any $\mathfrak{B}$-regular family $\mathcal{F}$ of biweights on $\mathfrak{A}$

(i) $\mathcal{D}\left(p_{\mathcal{F}}\right)=\mathcal{D}\left(q_{\mathcal{F}}\right)$ is a partial *-algebra in $\mathfrak{A}$;

(ii) $p_{\mathcal{F}}(a)=q_{\mathcal{F}}(a)$ for every $a \in \mathcal{D}\left(p_{\mathcal{F}}\right)$;

(iii) $p_{\mathcal{F}}$ is an unbounded $\mathrm{C}^{*}$-seminorm on $\mathcal{D}\left(p_{\mathcal{F}}\right)$.

3.2. $C^{*}$-seminorms and admissible biweights. Similarly to the case of positive linear functionals, the notion of admissibility can be introduced for a biweight $\varphi$ but it is linked to a core of admissibility.

DeFinition 13. A biweight $\varphi$ on the partial *-algebra is admissible if there exists a core $B(\varphi)$ such that

$$
\forall a \in \mathfrak{A}, \exists \gamma_{a}>0: \quad \varphi(a x, a x) \leq \gamma_{a} \varphi(x, x), \quad \forall x \in B(\varphi) .
$$

Assume now that $\mathcal{F}$ is a $\mathfrak{B}$-regular family of biweights with common core $\mathfrak{B}$. Let $\varphi \in \mathcal{F}, a \in \mathfrak{A}$ and $x \in \mathfrak{B}$. Then, if $\varphi(x, x)=0$, we get $\varphi(a x, a x)=0$. If $\varphi(x, x)>0$, then, putting $w=x / \varphi(x, x)^{1 / 2}$ we have $\varphi(w, w)=1$ and

$$
\varphi(a x, a x)=\varphi(a w, a w) \varphi(x, x) .
$$

Then if $a \in D\left(p_{\mathcal{F}}\right)$ we obtain

$$
\varphi(a x, a x) \leq p_{\mathcal{F}}(a)^{2} \varphi(x, x) .
$$

Then the following is clear:

Proposition 14. Let $\mathcal{F}$ be a $\mathfrak{B}$-regular family of biweights with common core $\mathfrak{B}$. If $D\left(p_{\mathcal{F}}\right)=\mathfrak{A}$, then each $\varphi \in \mathcal{F}$ is admissible.

Definition 15. Let $q$ be a seminorm defined on a partial ${ }^{*}$-subalgebra $\mathcal{D}(q)$ of $\mathfrak{A}$ and $\varphi$ a sesquilinear form on $\mathcal{D}(\varphi) \times \mathcal{D}(\varphi), \mathcal{D}(\varphi) \subseteq \mathfrak{A}$. We say that $\varphi$ is $q$-continuous if

(i) $\mathcal{D}(q) \subseteq \mathcal{D}(\varphi)$;

(ii) $\exists \gamma_{\varphi}>0: \quad|\varphi(a, b)| \leq \gamma_{\varphi} q(a) q(b), \quad \forall a, b \in \mathcal{D}(q)$.

We denote with $\|\varphi\|_{q}$ the infimum of all positive constants for which (ii) holds.

Let $\varphi$ be a biweight on $\mathfrak{A}$ with domain $\mathcal{D}(\varphi)$ and core $B(\varphi)$. For each $x \in \mathfrak{B}(\varphi)$, we define a positive sesquilinear form on $\mathfrak{A} \times \mathfrak{A}$ by

$$
\varphi_{x}(a, b)=\varphi(a x, b x), \quad a, b \in \mathfrak{A} .
$$


DeFinition 16. Let $\varphi$ be a biweight on $\mathfrak{A}$ with domain $\mathcal{D}(\varphi)$ and core $B(\varphi)$ and let $q$ be a seminorm with domain $\mathcal{D}(q)$. We say that $\varphi$ has a $q$-continuous $B(\varphi)$-orbit if each $\varphi_{x}$, $x \in B(\varphi)$, is $q$-continuous.

A biweight $\varphi$ could have a $q$-continuous $B(\varphi)$-orbit, without $\varphi$ being $q$-continuous.

For a fixed $\mathfrak{B} \subseteq R \mathfrak{A}$ we denote with $B W(\mathfrak{A}, \mathfrak{B})$ the family of all biweights of $\mathfrak{A}$ having $\mathfrak{B}$ as a core and we consider a seminorm $q$ on $\mathfrak{A}$ with $\mathcal{D}(q)=\mathfrak{A}$. Let

$$
C O(q, \mathfrak{B})=\{\varphi \in B W(\mathfrak{A}, \mathfrak{B}): \varphi \text { has a } q \text {-continuous } \mathfrak{B} \text {-orbit }\}
$$

and

$$
C O^{e}(q, \mathfrak{B})=\left\{\varphi \in C O(q, \mathfrak{B}):\left\|\varphi_{x}\right\|_{q} \leq \varphi(x, x), x \in \mathfrak{B}\right\} .
$$

The following characterizes admissibility of biweights.

TheOREM 17. Let $\varphi$ be a biweight on $\mathfrak{A}$ with domain $\mathcal{D}(\varphi)$. A necessary and sufficient condition for $\varphi$ to be admissible is that there exists an everywhere defined submultiplicative seminorm $q$ on $\mathfrak{A}$ and a core $B(\varphi)$ for $\varphi$ such that $\varphi \in C O^{e}(q, B(\varphi))$.

The next step consists in considering conditions under which $C O^{e}(q, \mathfrak{B})=C O(q, \mathfrak{B})$, like it happens for the corresponding objects on *-algebras. For doing this we recall some definitions.

If $\mathfrak{A}$ is a partial *-algebra and $a \in \mathfrak{A}$, the length $\ell(a)$ is the largest $n \in \mathbb{N} \cup\{\infty\}$ such that $a^{n}$ is well-defined in $\mathfrak{A}$.

We put

$$
R \mathfrak{A}_{\infty}=\left\{a \in R \mathfrak{A}: \ell\left(a^{*} a\right)=\infty\right\} .
$$

Now, let $q$ be a $\mathrm{C}^{*}$-seminorm on $\mathfrak{A}$ (i.e., $\mathcal{D}(q)=\mathfrak{A}$ ), $\mathfrak{B} \subseteq R \mathfrak{A}$ and $\varphi$ a biweight on $\mathfrak{A}$ with $\varphi \in C O(q, \mathfrak{B})$. Then a Kaplansky-like inequality can be proven for elements of $R \mathfrak{A}_{\infty}$. This implies that

$$
\left|\varphi_{x}(a, b)\right| \leq \varphi(x, x) q(a) q(b), \quad \forall a, b \in R \mathfrak{A}_{\infty} .
$$

If $R \mathfrak{A}_{\infty}$ is $q$-dense in $\mathfrak{A},(10)$ extends easily to the whole $\mathfrak{A}$. This, in turn implies that

Proposition 18. Let $q$ be a $C^{*}$-seminorm on $\mathfrak{A}$ and assume that $R \mathfrak{A}_{\infty}$ is $q$-dense in $\mathfrak{A}$. Then, for each $\mathfrak{B} \subset R \mathfrak{A}, C O(q, \mathfrak{B})=C O^{e}(q, \mathfrak{B})$.

The assumption that $R \mathfrak{A}$ is dense in $\mathfrak{A}$ implies [3, Lemma 8.1.2] that there exists a $\mathrm{C}^{*}$-algebra $\mathfrak{R}$, with \|\|$_{q}$, and a linear map $x \in \mathfrak{A} \rightarrow \widetilde{x} \in \mathfrak{R}$, preserving the involution and such that $\widetilde{x \cdot y}=\widetilde{x} \cdot \widetilde{y}$ whenever $x \cdot y$ is well defined. By the Gelfand-Naimark theorem, there exists an isometric ${ }^{*}$-isomorphism $\Phi$ of $\mathfrak{R}$ onto a $\mathrm{C}^{*}$-algebra $\mathfrak{M}$ of bounded operators in Hilbert space $\mathcal{H}$. If $\xi \in \mathcal{H}$, we put

$$
\varphi_{\xi}(a, b)=\langle\Phi(\widetilde{a}) \xi \mid \Phi(\widetilde{b}) \xi\rangle, \quad a, b \in \mathfrak{A} .
$$

Then $\varphi_{\xi}$ is a well-defined positive sesquilinear form on $\mathfrak{A} \times \mathfrak{A}$ and it can be shown that it is a biweight with $D\left(\varphi_{\xi}\right)=\mathfrak{A}$ and $B\left(\varphi_{\xi}\right)=\mathfrak{B}$. Moreover $\varphi_{\xi} \in C O^{e}(q, \mathfrak{B})$. Now, if $e \in \mathfrak{B}$, we have

$$
q(a)^{2}=\sup \left\{\varphi_{\xi}(a, a) ;\|\xi\|=1\right\} \leq p_{\mathcal{F}}(a)^{2}, \quad \forall a \in \mathfrak{A} .
$$

But $p_{\mathcal{F}}(a) \leq q(a)$, for every $a \in \mathfrak{A}$. Then 
TheOREM 19. Let $q$ be a $C^{*}$-seminorm on $\mathfrak{A}$ and $\mathfrak{B}$ a subspace of $R \mathfrak{A}, q$-dense in $\mathfrak{A}$ and such that $e \in \mathfrak{B}$. If $\mathcal{F}=C O(q, \mathfrak{B})$, then $\mathcal{D}\left(p_{\mathcal{F}}\right)=\mathfrak{A}$, and

$$
q(a)=p_{\mathcal{F}}(a), \quad \forall a \in \mathfrak{A} .
$$

4. The case of quasi *-algebras. The results of the previous section apply, of course, to the case of a quasi $*_{\text {-algebra }}\left(\mathfrak{A}, \mathfrak{A}_{0}\right)$. The particularly simple structure of quasi $*_{-}$ algebras allows however to obtain results similar to those of the previous section without making reference to the notion of biweight. We will briefly overview in this section the main results obtained in [22].

To begin with, we define certain particular types of seminorms on a quasi *-algebra.

Definition 20. Let $\left(\mathfrak{A}, \mathfrak{A}_{0}\right)$ be a quasi *-algebra with unit $e$ and $p$ a seminorm on $\mathfrak{A}$. We say that $p$ is a $\mathrm{Q}^{*}$-seminorm on $\left(\mathfrak{A}, \mathfrak{A}_{0}\right)$ if

$\left(\mathrm{Q}^{*} 1\right) p(a)=p\left(a^{*}\right), \quad \forall a \in \mathfrak{A}$;

$\left(\mathrm{Q}^{*} 2\right) p(e)=1$

$\left(\mathrm{Q}^{*} 3\right)$ for each $x \in \mathfrak{A}_{0}$ there exists $\gamma_{x}>0$ such that

$$
p(a x) \leq \gamma_{x} p(a), \quad \forall a \in \mathfrak{A} .
$$

If $p$ is a $\mathrm{Q}^{*}$-seminorm, we can define

$$
p_{0}(x):=\max \left\{\sup _{p(a)=1} p(a x), \sup _{p(a)=1} p(x a)\right\},
$$

then $p(x) \leq p_{0}(x)$ for every $x \in \mathfrak{A}_{0}$ and

$$
p(a x) \leq p(a) p_{0}(x), \quad \forall a \in \mathfrak{A}, x \in \mathfrak{A}_{0} .
$$

We will call $p_{0}$ the reduced seminorm of $p$.

One of the most favorable situations occurs when, in analogy to what happens for the so-called $\mathrm{CQ}^{*}$-algebras $[6], p_{0}$ is a $\mathrm{C}^{*}$-seminorm on $\mathfrak{A}_{0}$.

Definition 21. A $\mathrm{Q}^{*}$-seminorm $p$ is called a $\mathrm{CQ}^{*}$-seminorm if $p_{0}$ is a $\mathrm{C}^{*}$-seminorm on $\mathfrak{A}_{0}$.

If $p$ itself satisfies the $\mathrm{C}^{*}$-condition when restricted to $\mathfrak{A}_{0}$, then we call it an extended $\mathrm{C}^{*}$-seminorm on $\left(\mathfrak{A}, \mathfrak{A}_{0}\right)$.

Definition 22. Let $\left(\mathfrak{A}, \mathfrak{A}_{0}\right)$ be a quasi *-algebra with unit $e$. A positive sesquilinear form $\varphi$ on $\mathfrak{A} \times \mathfrak{A}$ is called left invariant if

$$
\varphi(x a, b)=\varphi\left(a, x^{*} b\right), \quad \forall a, b \in \mathfrak{A}, x \in \mathfrak{A}_{0} .
$$

The set of all positive, left invariant sesquilinear forms is denoted with $\mathcal{P}_{\ell}$.

Due to positivity, any $\varphi \in \mathcal{P}_{\ell}$ is hermitian, i.e. $\varphi(b, a)=\overline{\varphi(a, b)}$ for any $a, b \in \mathfrak{A}$, and satisfies the Cauchy-Schwarz inequality.

In analogy to the cases of states or biweights, an element $\varphi \in \mathcal{P}_{\ell}$ is called admissible if for each $a \in \mathfrak{A}$ there exists $\gamma_{a}>0$ such that

$$
\varphi(a x, a x) \leq \gamma_{a} \varphi(x, x), \quad \forall x \in \mathfrak{A}_{0} .
$$


If $\varphi \in \mathcal{P}_{\ell}$ and $a \in \mathfrak{A}$, we can define a positive linear functional $\omega_{\varphi}^{a}$ on $\mathfrak{A}_{0}$ by

$$
\omega_{\varphi}^{a}(x)=\varphi(x a, a), \quad x \in \mathfrak{A}_{0} .
$$

Let $\mathcal{F}$ be any subset of $\mathcal{P}_{\ell}$. We put:

$$
\mathcal{F}^{0}=\left\{\omega_{\varphi}^{a}: \varphi \in \mathcal{F}, a \in \mathfrak{A}\right\} .
$$

Then $\mathcal{F}^{0}$ is balanced [Definition 2]; thus the set

$$
\mathfrak{A}_{0}\left(\mathcal{F}^{0}\right)=\left\{x \in \mathfrak{A}_{0}: \sup \left\{\omega_{\varphi}^{a}\left(x^{*} x\right): \varphi \in \mathcal{F}, a \in \mathfrak{A}, \varphi(a, a)=1\right\}<\infty\right\}
$$

is a *-subalgebra of $\mathfrak{A}_{0}$ and

$$
|x|_{\mathcal{F}^{0}}=\left(\sup \left\{\omega_{\varphi}^{a}\left(x^{*} x\right): \varphi \in \mathcal{F}, a \in \mathfrak{A}, \varphi(a, a)=1\right\}\right)^{1 / 2}
$$

defines a $\mathrm{C}^{*}$-seminorm on $\mathfrak{A}_{0}\left(\mathcal{F}^{0}\right)$.

Let $\mathcal{F} \subseteq \mathcal{P}_{\ell}$. For $a \in \mathfrak{A}$, we put

$$
p_{\mathcal{F}}^{R}(a)=\sup _{\varphi \in \mathcal{F}_{s}} \varphi(a, a)^{1 / 2}, \quad \text { where } \quad \mathcal{F}_{s}=\{\varphi \in \mathcal{F}: \varphi(e, e)=1\} .
$$

Then, we set

$$
\begin{aligned}
\mathfrak{A}(\mathcal{F})= & \left\{a \in \mathfrak{A}: p_{\mathcal{F}}^{R}(a)<\infty \text { and } p_{\mathcal{F}}^{R}\left(a^{*}\right)<\infty\right\} \\
& p_{\mathcal{F}}(a)=\max \left\{p_{\mathcal{F}}^{R}(a), p_{\mathcal{F}}^{R}\left(a^{*}\right)\right\}
\end{aligned}
$$

Then $\mathfrak{A}(\mathcal{F})$ is a ${ }^{*}$-invariant subspace of $\mathfrak{A}$ but, in general, need not be quasi ${ }^{*}$-algebra over $\mathfrak{A}_{0}\left(\mathcal{F}^{0}\right)$. There is, however, some special situation.

If $\varphi \in \mathcal{P}_{\ell}$ and $x \in \mathfrak{A}_{0}$, we put $\varphi_{x}(a, b):=\varphi(a x, b x)$. It is easily seen that $\varphi_{x} \in \mathcal{P}_{\ell}$.

Definition 23. Let $\mathcal{F} \subseteq \mathcal{P}_{\ell}$. We say that $\mathcal{F}$ is strongly balanced if, for each $\varphi \in \mathcal{F}$ and for each $x \in \mathfrak{A}_{0}$, the following conditions are fulfilled:

(i) $\varphi_{x} \in \mathcal{F}$;

(ii) if $\varphi(x, x)=0$ for some $x \in \mathfrak{A}_{0}$, then $\varphi(a x, a x)=0$ for any $a \in \mathfrak{A}$,

If $\mathcal{F}$ be strongly balanced, then the following inequality holds

$$
p_{\mathcal{F}}^{R}(a x) \leq|x|_{\mathcal{F}^{0}} \cdot p_{\mathcal{F}}^{R}(a), \quad \forall a \in \mathfrak{A}(\mathcal{F}), x \in \mathfrak{A}_{0}\left(\mathcal{F}^{0}\right)
$$

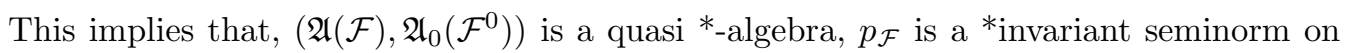
$\left(\mathfrak{A}(\mathcal{F}), \mathfrak{A}_{0}\left(\mathcal{F}^{0}\right)\right)$ and

$$
p_{\mathcal{F}}(a x) \leq|x|_{\mathcal{F}^{0}} \cdot p_{\mathcal{F}}(a), \quad \forall a \in \mathfrak{A}(\mathcal{F}), x \in \mathfrak{A}_{0}\left(\mathcal{F}^{0}\right)
$$

A family $\mathcal{F} \subseteq \mathcal{P}_{\ell}$ is called well-behaved if

$$
p_{\mathcal{F}}(a)=p_{\mathcal{F}}^{R}(a)=p_{\mathcal{F}}^{R}\left(a^{*}\right), \quad \forall a \in \mathfrak{A}
$$

Following the different assumptions on $\mathcal{F}$, we then have

Proposition 24. Let $\left(\mathfrak{A}, \mathfrak{A}_{0}\right)$ be a quasi ${ }^{*}$-algebra and $\mathcal{F} \subset \mathcal{P}_{\ell}$ a family of sesquilinear forms on $\mathfrak{A} \times \mathfrak{A}$. Then the following statements hold:

(i) There exists a quasi ${ }^{*}$-algebra $\left(\mathfrak{A}(\mathcal{F}), \mathfrak{A}_{0}\left(\mathcal{F}^{0}\right)\right)$ contained in $\left(\mathfrak{A}, \mathfrak{A}_{0}\right)$ such that $p_{\mathcal{F}}$ is $a{ }^{*}$-invariant seminorm on $\left(\mathfrak{A}(\mathcal{F}), \mathfrak{A}_{0}\left(\mathcal{F}^{0}\right)\right)$.

(ii) If $\mathcal{F}$ is well-behaved, then $p_{\mathcal{F}}$ is a $Q^{*}$-seminorm on $\left(\mathfrak{A}(\mathcal{F}), \mathfrak{A}_{0}\left(\mathcal{F}^{0}\right)\right)$.

(iii) If $\mathcal{F}$ is strongly balanced, then $p_{\mathcal{F}}$ is an extended $C^{*}$-seminorm on $\left(\mathfrak{A}(\mathcal{F}), \mathfrak{A}_{0}\left(\mathcal{F}^{0}\right)\right)$. 
Of course, if ||$_{\mathcal{F}^{0}}=\left(p_{\mathcal{F}}\right)_{0}$, where $\left(p_{\mathcal{F}}\right)_{0}$ is the reduced seminorm of $p_{\mathcal{F}}$ [see (12)] then $p_{\mathcal{F}}$ would automatically be a $\mathrm{CQ}^{*}$-seminorm.

Proposition 25. Let $\mathcal{F} \subset \mathcal{P}_{\ell}$ be a well-behaved family of sesquilinear forms and $\left(\mathfrak{A}(\mathcal{F}), \mathfrak{A}_{0}\left(\mathcal{F}^{0}\right)\right)$ the quasi ${ }^{*}$-algebra constructed as above. The following statements are equivalent:

(i) $|x|_{\mathcal{F}^{0}}=\left(p_{\mathcal{F}}\right)_{0}(x), \quad \forall x \in \mathfrak{A}_{0}\left(\mathcal{F}^{0}\right)$.

(ii) $\varphi(x a, x a) \leq\left(p_{\mathcal{F}}\right)_{0}(x)^{2} \varphi(a, a), \quad \forall \varphi \in \mathcal{F}, x \in \mathfrak{A}_{0}, a \in \mathfrak{A}$.

(iii) For each $\varphi \in \mathcal{F}$ and $a \in \mathfrak{A}, \omega_{\varphi}^{a}$ is $\left(p_{\mathcal{F}}\right)_{0}$-continuous.

If any of the previous statements holds then $p_{\mathcal{F}}$ is a $C Q^{*}$-seminorm on $\left(\mathfrak{A}(\mathcal{F}), \mathfrak{A}_{0}\left(\mathcal{F}^{0}\right)\right)$.

EXAMPLE 2. Let $I$ be a compact interval on the real line and consider the quasi*-algebra [7] of functions $\left(L^{p}(I), C(I)\right)$ where $C(I)$ stands for the ${ }^{*}$-algebra of all continuous functions on $I$ and $L^{p}(I)$ is the usual $L^{p}$-space on $I$. We assume that $p \geq 2$. Let $w \in L^{\frac{p}{p-2}}(I)$ (we take $1 / 0=\infty$ ) and $w \geq 0$. Then

$$
\varphi^{(w)}(f, g)=\int_{I} f(x) \overline{g(x)} w(x) d x, \quad f, g \in L^{p}(I)
$$

defines a left invariant positive sesquilinear form on $L^{p}(I)$. If $w \in L^{\infty}(I)$, then $\varphi^{(w)}$ is admissible. We put

$$
\mathcal{F}=\left\{\varphi^{(w)}: w \in L^{\frac{p}{p-2}}(I), w \geq 0\right\} .
$$

It is easy to see that $\mathcal{F}$ is strongly balanced and that $\varphi^{(w)} \in \mathcal{F}_{s}$ if, and only if, $\|w\|_{1}=1$. Very easy estimates show that $\mathfrak{A}_{0}\left(\mathcal{F}^{0}\right)=C(I)$ and $|\phi|_{\mathcal{F}^{0}}=\|\phi\|_{\infty}$. On the other hand,

$$
\mathfrak{A}(\mathcal{F})=\left\{f \in L^{p}(I): \sup _{\|w\|_{1}=1} \int_{I}|f(x)|^{2} w(x) d x<\infty\right\}=L^{\infty}(I) .
$$

Therefore, the extended $\mathrm{C}^{*}$-seminorm $p_{\mathcal{F}}$ coincides with the $L^{\infty}$-norm on $L^{\infty}(I)$.

ExAmple 3. Let $\mathcal{D}$ be a dense domain in Hilbert space $\mathcal{H}$ and let $\mathcal{L}^{\dagger}(\mathcal{D}, \mathcal{H})$ the weak partial *-algebra of operators defined in the Introduction. Let

$$
\mathcal{B}(\mathcal{D})=\left\{X \in \mathcal{L}^{\dagger}(\mathcal{D}, \mathcal{H}): X \text { is bounded and } X: \mathcal{D} \rightarrow \mathcal{D}, X^{\dagger}: \mathcal{D} \rightarrow \mathcal{D}\right\} .
$$

Then $\mathcal{B}(\mathcal{D})$ is a ${ }^{*}$-algebra and $\left(\mathcal{L}^{\dagger}(\mathcal{D}, \mathcal{H}), \mathcal{B}(\mathcal{D})\right)$ can be viewed as a quasi-*-algebra under the weak multiplication:

$$
A \square B f=A B f ; \quad B \square A f=\bar{B} A f, \quad A \in \mathcal{L}^{\dagger}(\mathcal{D}, \mathcal{H}), B \in \mathcal{B}(\mathcal{D}) .
$$

If $f \in \mathcal{D}$, the positive sesquilinear form $\varphi^{f}$ defined by

$$
\varphi^{f}(A, B)=<A f, B f>, \quad A, B \in \mathcal{L}^{\dagger}(\mathcal{D}, \mathcal{H})
$$

is left invariant.

Let now $\mathcal{M}$ be a subspace of $\mathcal{D}$ and let

$$
\mathcal{F}=\left\{\varphi^{f} ; f \in \mathcal{M}\right\}
$$

Then $\mathfrak{A}_{0}\left(\mathcal{F}^{0}\right)=\mathcal{B}(\mathcal{D})$ and $|B|_{\mathcal{F}^{0}}=\|B\|$, for each $B \in \mathcal{B}(\mathcal{D})$. On the other hand

$$
p_{\mathcal{F}}^{R}(A)=\sup _{\varphi \in \mathcal{F}_{s}} \varphi(A, A)^{1 / 2}=\| A\lceil\mathcal{M} \|
$$


Thus

$$
\mathfrak{A}(\mathcal{F})=\left\{A \in \mathcal{L}^{+}(\mathcal{D}, \mathcal{H}): A\left\lceil\mathcal{M} \text { and } A^{*}\lceil\mathcal{M} \text { are bounded }\}\right.\right.
$$

The corresponding seminorm

$$
p_{\mathcal{F}}(A)=\max \left\{\| A\left\lceil\mathcal{M}\|,\| A^{*}\lceil\mathcal{M} \|\}\right.\right.
$$

is a ${ }^{*}$-invariant seminorm but not, in general, a $\mathrm{Q}^{*}$-seminorm. If $\mathcal{M}=\mathcal{D}$, then $\mathfrak{A}(\mathcal{F})=$ $\mathfrak{A}_{0}\left(\mathcal{F}^{0}\right)=\mathcal{B}(\mathcal{D})$ and $p_{\mathcal{F}}$ is a $\mathrm{C}^{*}$-seminorm.

As we did for partial *-algebras, we can now examine the problem of the relationship between admissibility of a sesquilinear form $\varphi \in \mathcal{P}_{\ell}$ and its continuity with respect to some seminorm on $\left(\mathfrak{A}, \mathfrak{A}_{0}\right)$. This can be done in complete analogy to the case of partial *-algebras, but proofs are different. We do not enter the details, referring to [22] for more information. We simply give the main results.

Let $q$ be a $\mathrm{Q}^{*}$-seminorm on $\left(\mathfrak{A}, \mathfrak{A}_{0}\right)$ and we denote with $\mathcal{C}(q)$ the set of all $q$-continuous elements of $\mathcal{P}_{\ell}$. It is easy to see that for each $\Phi \in \mathcal{C}(q)$, then $\Phi_{x} \in \mathcal{C}(q)$ for every $x \in \mathfrak{A}_{0}$ (but $\mathcal{C}(q)$ is not necessarily strongly balanced, since (i) of Definition 23 may fail). Moreover one has $\left\|\Phi_{x}\right\|_{q} \leq\|\Phi\|_{q} q(x)^{2}$ for each $\Phi \in \mathcal{C}(q)$ and $x \in \mathfrak{A}_{0}$. As seen above, $\mathfrak{A}(\mathcal{C}(q))$ is a quasi *-algebra over $\mathfrak{A}_{0}\left(\mathcal{C}(q)^{0}\right)$ and $\mathfrak{A}_{0}\left(\mathcal{C}(q)^{0}\right)=\mathfrak{A}_{0}$.

In order to describe admissibility, we consider some special subset of $C(q)$. Let

$$
\mathcal{C}^{0}(q)=\left\{\varphi \in \mathcal{C}(q):\|\varphi\|_{q}=\varphi(e, e)\right\}
$$

and

$$
\mathcal{C}^{e}(q)=\left\{\varphi \in \mathcal{C}^{0}(q): \varphi_{x} \in \mathcal{C}^{0}(q), \forall x \in \mathfrak{A}_{0}\right\} .
$$

Let $\mathcal{F} \subset \mathcal{C}^{0}(q)$. In this case we have:

$$
p_{\mathcal{F}}^{R}(a)=\sup _{\varphi \in \mathcal{F}_{s}} \varphi(a, a)^{1 / 2} \leq q(a), \quad \forall a \in \mathfrak{A} .
$$

So $\mathfrak{A}(\mathcal{F})=\mathfrak{A}$ and therefore $p_{\mathcal{F}}$ is a ${ }^{*}$-invariant seminorm on $\mathfrak{A}$ (but not necessarily a $\mathrm{Q}^{*}$-seminorm). Moreover,

$$
p_{\mathcal{F}}(a) \leq p_{\mathcal{C}^{0}(q)}(a) \leq q(a), \quad \forall a \in \mathfrak{A} .
$$

From the definition itself it follows that if $\varphi \in \mathcal{C}^{e}(q)$, then $\varphi$ is admissible, i.e. $\mathcal{C}^{e}(q) \subset$ $\mathcal{P}_{\ell}^{a}$. This implies that $\mathcal{C}^{e}(q)$ is strongly balanced and therefore, $p_{\mathcal{C}^{e}(q)}$ is an extended $\mathrm{C}^{*}$-seminorm.

Proposition 26. Let $\varphi \in \mathcal{P}_{\ell}$. A necessary and sufficient condition for $\varphi$ to be admissible is that there exists an (everywhere defined) extended $C^{*}$-seminorm $q$ on $\left(\mathfrak{A}, \mathfrak{A}_{0}\right)$ such that $\varphi \in \mathcal{C}^{e}(q)$.

The discussion so far applies to the particular case where $q=p_{\mathcal{F}}$ for some subset $\mathcal{F}$ of $\mathcal{P}_{\ell}$. In this case, each $\varphi \in \mathcal{F}$ is $p_{\mathcal{F}}$-continuous on $\left(\mathfrak{A}(\mathcal{F}), \mathfrak{A}_{0}\left(\mathcal{F}^{0}\right)\right)$; i.e., $\mathcal{F} \subseteq \mathcal{C}\left(p_{\mathcal{F}}\right)$. If $\mathcal{F}$ is strongly balanced, we get:

$$
p_{\mathcal{F}}(a)=p_{\mathcal{C}^{e}\left(p_{\mathcal{F}}\right)}(a)=p_{\mathcal{C}^{0}\left(p_{\mathcal{F}}\right)}(a), \quad \forall a \in \mathfrak{A}
$$

and by Proposition 26, each $\varphi \in \mathcal{F}$ is admissible on $\left(\mathfrak{A}(\mathcal{F}), \mathfrak{A}_{0}\left(\mathcal{F}^{0}\right)\right.$. In other words, given a strongly balanced subset $\mathcal{F}$ of $\mathcal{P}_{\ell}$, it is always possible to find a quasi*-algebra contained in $\left(\mathfrak{A}, \mathfrak{A}_{0}\right)$, namely, $\left(\mathfrak{A}(\mathcal{F}), \mathfrak{A}_{0}\left(\mathcal{F}^{0}\right)\right)$, where $\varphi \in \mathcal{F}$ is admissible. 
EXAMPLE 4. Let $\mathcal{H}$ be a Hilbert space and let $\mathfrak{A} \subseteq \mathcal{B}(\mathcal{H})$ be any *-algebra with unit of bounded operators. If $f \in \mathcal{H}$, we put:

$$
\varphi^{f}(A, B)=<A f, B f>, \quad A, B \in \mathfrak{A} .
$$

Then each $\varphi^{f}$ is left-invariant and the set $\mathcal{F}=\left\{\varphi^{f} ; f \in \mathcal{H}\right\}$ is strongly balanced. In this case $\mathfrak{A}(\mathcal{F})=\mathfrak{A}_{0}\left(\mathcal{F}^{0}\right)=\mathfrak{A}$ and

$$
p_{\mathcal{F}}(A)=\|A\|, \quad A \in \mathfrak{A} .
$$

The set $\mathcal{C}\left(p_{\mathcal{F}}\right)$ consists of all sesquilinear forms $\Phi$ for which there exists $K>0$ such that:

$$
\Phi(a, b) \leq K\|A\|\|B\|, \quad \forall a, B \in \mathfrak{A} .
$$

This set properly contains $\mathcal{F}$, in general. Since $\mathfrak{A}$ is a ${ }^{*}$-algebra, $\mathcal{C}\left(p_{\mathcal{F}}\right)=\mathcal{C}^{0}\left(p_{\mathcal{F}}\right)=\mathcal{C}^{e}\left(p_{\mathcal{F}}\right)$ and $p_{\mathcal{F}}(A)=p_{\mathcal{C}}(A)=\|A\|$, for every $A \in \mathfrak{A}$.

In order to show that, in general, $\mathcal{F} \neq \mathcal{C}^{e}\left(p_{\mathcal{F}}\right)$ let us consider a bounded self-adjoint operator $A$ with continuous spectrum $\sigma \subset \mathbb{R}$ and let $C(\sigma)$ denote the ${ }^{*}$-algebra of all continuous functions on the compact set $\sigma$ with its usual sup norm \|\|$_{\infty}$. Let

$$
\mathfrak{A}=\{f(A) ; f \in C(\sigma)\}
$$

where $f(A)$ is defined via the functional calculus. As is known, each $f(A)$ is bounded and $\|f(A)\|=\|f\|_{\infty}$. Then $\mathfrak{A}$ is a $\mathrm{C}^{*}$-algebra of bounded operators. We take $\mathcal{F}$ as above.

Let now $\lambda_{0} \in \sigma$ be fixed. We define a sesquilinear form $\varphi_{\lambda_{0}}$ by

$$
\varphi_{\lambda_{0}}(f(A), g(A))=f\left(\lambda_{0}\right) \overline{g\left(\lambda_{0}\right)}, \quad f, g \in C(\sigma) .
$$

$\varphi_{\lambda_{0}}$ is positive, left invariant and bounded. Indeed,

$$
\left|\varphi_{\lambda_{0}}(f(A), g(A))\right|=\left|f\left(\lambda_{0}\right) \overline{g\left(\lambda_{0}\right)}\right| \leq\|f\|_{\infty}\|g\|_{\infty}=\|f(A)\|\|g(A)\|, \quad f, g \in C(\sigma) .
$$

This implies that $\left\|\varphi_{\lambda_{0}}\right\|_{p_{\mathcal{F}}} \leq 1$. In fact, it is easy to realize that the equality holds true. We now show that it is not possible to find $\eta \in \mathcal{H}$ such that

$$
\varphi_{\lambda_{0}}(f(A), g(A))=<f(A) \eta, g(A) \eta>, \quad \forall f, g \in C(\sigma) .
$$

Indeed, if $E(\cdot)$ denotes the spectral measure of $A$, we would have:

$$
\begin{aligned}
\varphi_{\lambda_{0}}(f(A), g(A)) & =<f(A) \eta, g(A) \eta> \\
& =\int_{\sigma} f(\lambda) \overline{g(\lambda)} d<E(\lambda) \eta, \eta>=f\left(\lambda_{0}\right) \overline{g\left(\lambda_{0}\right)}, \quad \forall f, g \in C(\sigma),
\end{aligned}
$$

and this is possible only if $\lambda_{0}$ is an eigenvalue of $A$.

Conclusion. The discussion so far has been focused on the problem of construction of Gelfand-like seminorms in partial or quasi *-algebras. Of course, one expects that they can provide useful information on these structures, especially when the latter carry some locally convex topology. The first step in this direction is to consider the case of normed or even Banach partial *-algebras that have been recently studied in [4]. Therein, in fact Gelfand-like seminorms defined by biweights having a common core are used to introduce notions (like that of *-radical) that could hardly be defined (due to the partial nature of the multiplication) by a simple adaptation of the ordinary definitions. Some interesting results on the structure of Banach partial *-algebras have been obtained (for instance, on the continuity of ${ }^{*}$-representations). Even if these results can only be considered as 
preliminary (and work is still in progress!), they seem to motivate sufficiently the study of the extensions of $\mathrm{C}^{*}$-seminorms to the partial algebraic case.

\section{References}

[1] J. Alcantara and J. Yngvason, Algebraic quantum field theory and noncommutative moment problem I, Ann. Inst. Henri Poincaré 48 (1988), 147-159.

[2] J.-P. Antoine, A. Inoue and C. Trapani, Biweights on partial *algebras, J. Math. Anal. Appl. 242 (2000), 164-190.

[3] J.-P. Antoine, A. Inoue and C. Trapani, Partial *algebras and Their Operator Realizations, Kluwer, Dordrecht, 2002.

[4] J.-P. Antoine and C. Trapani, Banach partial *-algebras: basic properties, representations, spectrum, preprint, Palermo/Louvain-la-Neuve, 2003.

[5] F. Bagarello, A. Inoue and C. Trapani, Unbounded $C^{*}$-seminorms on partial *algebras, Zeit. Anal. Anwen. 20 (2001), 295-314.

[6] F. Bagarello and C. Trapani, CQ*-algebras: structure properties, Publ. RIMS, Kyoto Univ. 32 (1996), 85-116.

[7] F. Bagarello and C. Trapani, $L^{p}$-spaces as quasi *algebras, J.Math. Anal. Appl. 197 (1996), 810-824.

[8] S. J. Bhatt, A. Inoue and K.-D. Kürsten, Well-behaved unbounded operator representations and unbounded $C^{*}$-seminorms, J. Math. Soc. Japan (to appear).

[9] S. J. Bhatt, A. Inoue and H. Ogi, On $C^{*}$-spectral algebras, International Workshop on Operator Theory (Cefalù, 1997), Rend. Circ. Mat. Palermo (2) Suppl. 1998, no. 56, 207213.

[10] S. J. Bhatt, A. Inoue and H. Ogi, Admissibility of weights on non-normed *algebras, Trans. American Math. Soc. 351 (1999), 183-208.

[11] S. J. Bhatt, A. Inoue and H. Ogi, Unbounded $C^{*}$-seminorms on *-algebras, in: Proceedings of the Second ISAAC Congress, Vol. 2 (Fukuoka, 1999), 863-870, Int. Soc. Anal. Appl. Comput., 8, Kluwer Acad. Publ., Dordrecht, 2000.

[12] S. J. Bhatt, A. Inoue and H. Ogi, Unbounded $C^{*}$-seminorms and unbounded $C^{*}$-spectral algebras, J. Operator Theory 45 (2001), 53-80.

[13] F. F. Bonsall and J. Duncan, Complete Normed Algebras, Springer, Berlin, 1973.

[14] R. S. Doran and V. A. Belfi, Characterization of $C^{*}$-algebras, Dekker, New York, Basel 1986.

[15] E. G. Effros, A decomposition theory for representations of $C^{*}$-algebras, Trans. Amer. Math. Soc. 107 (1963), 83-106.

[16] J. M. G. Fell, The structure of algebras of operator fields, Acta Math. 106 (1961), 233-280.

[17] G. Lassner, Topological algebras and their applications in quantum statistics, Wiss. Z. KMU Leipzig Math.-Nat. R. 6 (1981), 572-595.

[18] G. Lassner, Algebras of unbounded operators and quantum dynamics, Physica 124A (1984), 471-480.

[19] K. Schmüdgen, The order structure of of topological *-algebras of unbounded operators I, Rep. Math. Phys. 7 (1975), 215-227.

[20] K. Schmüdgen, Unbounded Operator Algebras and Representation Theory, Birkhäuser, Basel, 1990. 
[21] Z. Sebestyén, Every $C^{*}$-seminorm is automatically submultiplicative, Period. Math. Hungar. 10 (1979), 1-8.

[22] C. Trapani, Some seminorms on quasi *-algebras, Studia Matematica 158 (2003), 99 -115.

[23] C. Trapani and F. Tschinke, Unbounded $C^{*}$-seminorms and biweights on partial ${ }^{*}$-algebras, preprint, Dipartimento di Matematica ed Applicazioni n. 201, Palermo, 2003.

[24] J. Yngvason, Algebraic quantum field theory and noncommutative moment problem II, Ann. Inst. Henri Poincaré 48 (1988), 161-173.

[25] B. Yood, C*-seminorms, Studia Mathematica 118 (1996), 19-26. 